\title{
A Transmit Diversity Technique for OFDM System
}

\author{
Hema Thota ${ }^{1}$, Ch.Santhi Rani ${ }^{2}$, Kiranmayee Kagitha ${ }^{3}$, Anusha Balagam ${ }^{4}$, Anusha Goriparthi ${ }^{5}$, \\ Naga Lakshmi Devarapalli ${ }^{6}$ \\ B.Tech, Electronics and Communication Engineering, D.M.S S.V.H College of Engg., Machilipatnam, India 1, 3, 4, 5, 6 \\ Professor, Electronics and Communication Engg., D.M.S S.V.H College of Engg., Machilipatnam, India ${ }^{2}$
}

\begin{abstract}
In current 4G systems growing demand of multimedia services and the growth of internet related contents lead to increasing interest to high speed communications. One indispensable problem of the wireless channel is fading, which can be mitigated by diversity. There are various coding methods to achieve high reliability for Multiple Input Multiple Output- Orthogonal Frequency Division Multiplexing (MIMO-OFDM) systems earlier. Recently, Space Time Block Codes (STBC) have gained much attention as an effective transmit diversity technique to provide reliable transmission with high peak data rates to increase the speed and performance of wireless communication systems. This paper presents the performance evaluation of OFDM system, in which fading is reduced by using diversity techniques along with coding methods under various fading channels such as Rician and Rayleigh fading channels. In this work, a performance criterion such as Bit Error Rate (BER) for low Signal to Noise Ratio (SNR) ranging from 10dB to 20dB is analyzed. The BER for OFDM system without including STBC is $1.776 \times 10^{-3}$ and including STBC is $1.621 \times 10^{-3}$ over Rician Channel. Whereas, over Rayleigh channel the BER for OFDM system without including STBC is $1.834 \times 10^{-5}$ and including STBC, it is reduced to $6.345 \times 10^{-6}$ for the same SNR of $14 \mathrm{~dB}$. Thus, OFDM system with STBC provide high data rate with good reliability.
\end{abstract}

Keywords: Wireless channel, MIMO, OFDM, BER, STBC, SNR, LoS

\section{INTRODUCTION}

In a world of fast changing technology, there is a rising promising system configurations that is adopted for requirement for people to communicate and get connected standards such as Long Term Evolution (LTE) developed with each other to have appropriate and timely access to by $3^{\text {rd }}$ Generation Partnership Project (3GPP).

information regardless of the location of the each The propagation of radio waves through the atmosphere individual. The increasing demands and requirements for including the ionosphere is not a simple phenomenon to wireless communication systems ubiquity have led to the model. Atmospheric propagation can show a wide range of need for the better fundamental issues in communication behaviors based on factors like frequency, bandwidth of theory and their implications for the design of highly- the signal, types of antennas used, terrain and weather capable wireless systems. High transmission rate and high conditions. The presence of these paths is due to performance are the challenging requirements of future atmospheric reflections, refractions and scattering. In a wireless broadband communications. In a multipath multipath fading environment if a Line of Sight (LoS) wireless channel environment, the deployment of Multiple component is available then the channel is referred to as a Input Multiple Output (MIMO) systems leads to the Rician channel. On the other hand if there is no LoS achievement of high data rate transmission and to radically component then the channel is referred to as a Rayleigh improve link reliability without increasing the total fading channel.

transmission power or bandwidth.

An effective and practical way to approach the $A$ requirements of MIMO wireless channels is to employ This paper is organized into five sections where, section I space time block coding in which data is coded through gives a brief introduction, section II deals about MIMOspace and time to improve the reliability of the OFDM approach, Section III gives about the space time transmission, as redundant copies of the original data are block coding and channel model that has been taken into sent over independent fading channels. Then all the signal consideration, while simulation results and conclusions are copies are combined at the receiver in an optimal way to made in sections IV and V respectively.

extract as much information from each of them as possible. OFDM can reduce the effect of frequency selective channel. This is because OFDM is a multi-carrier transmission technique, which divides the available spectrum into many carriers, each one being modulated by a low-rate data stream. One popular combination of MIMO and OFDM is the STBC-OFDM. Furthermore, this work is motivated from such STBC-OFDM system. In this context, the STBC-OFDM system is one of most

\section{B. $\quad$ Literature Survey}

Although, the studies and systems based on the concept of Orthogonal Frequency Division Multiplexing (OFDM) have been publishing since 1958, most of the applications using current form of OFDM were developed during 1980s and 90s. However, there is still a lot of research on increasing the data rate. 
Hussain A. Alhassan, Dr.Eman Abdel Fattah [1] evaluated the performance of IEEE 802.16- 2004 and they found that the rate of data transmission is being increased.

G.J.Foschini and M.Gans [2] offer the system with high data rate transmission without increasing the total transmission power or Bandwidth.

Md.Mejabaul Haquel at all [3] Analyzed the performance of two transmit antennas with more receive antennas is much better than that of the system with two transmit antenna and less receive antennas in term of BER due to the more diversity gain of Alamouti's code.

V.Tarokh, Z.Liu at all [4] and [5] mentioned how data is coded through space and time to improve the reliability of the transmission, as redundant copies of the original data are sent over independent fading channels. Whereas this paper discusses how Space Time Block Codes are used to reduce BER

\section{MIMO - OFDM SYSTEM}

OFDM is an efficient technique for transmitting data over frequency selective channels. The main idea behind OFDM is to divide a broadband frequency channel in to a few narrowband sub-channels. Then, each sub-channel is a flat fading channel despite the frequency selective nature of the broadband channel.

The orthogonality condition of the two signals in OFDM can be given by

$\int_{F} S_{i}(f, t) S_{j}^{*}(f, t) d t=\left\{\begin{array}{l}1, i=j \\ 0, i \neq j\end{array} ; i, j=1,2 \ldots \ldots k\right.$

To generate these parallel sub-carriers in OFDM, an Inverse Fast Fourier Transform (IFFT) is applied to a block of $L$ data symbols. To avoid Inter Symbol Interference (ISI) due to the channel delay spread, a few Cyclic Prefix (CP) symbols are inserted in the block. The cyclic prefix samples are also called guard intervals. Basically, the last $g$ samples of the block are duplicated in front of the block as the cyclic prefix. The number of these cyclic prefix samples, should be bigger than the length of the channel impulse response. The effects of the cyclic prefix samples eliminate ISI and convert the convolution between the transmit symbols and the channel to a circular convolution. These cyclic prefix samples are removed at the receiver. Then, a Fast Fourier Transform (FFT) is utilized at the receiver to recover the block of $L$ received symbols. The Fig. 1 shows modulation and demodulation steps at OFDM transmitter and receiver. This paper presents OFDM system designed for MIMO channels. This is usually called MIMO-OFDM.
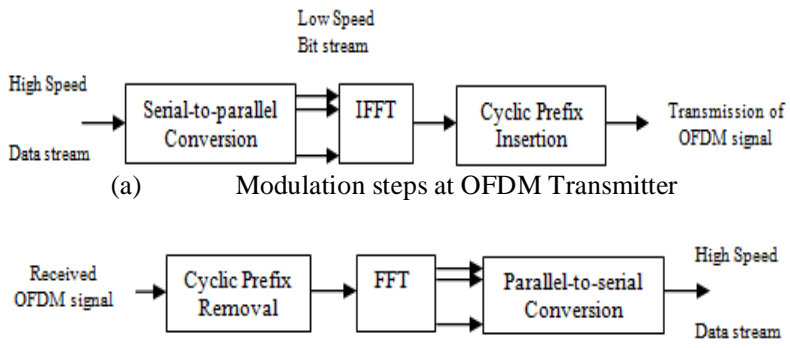

(b) Demodulation steps at OFDM Receiver

Fig.1 Block diagram of OFDM system.

\section{STBC FOR OFDM SYSTEM AND CHANNEL MODELS}

A. $\quad$ Space Time Block Codes

In this paper, space-time block codes are presented and the performance of MIMO fading channels is evaluated by simulations. The Alamouti code is one of the block codes, which is a simple two branch transmit diversity scheme. The key features of the scheme is orthogonality between the sequences generated by two transmit antennas. The space time block codes can achieve the full transmit diversity specified by the number of the transmit antennas $\mathrm{n}_{\mathrm{T}}$, while allowing a very simple maximum-likelihood decoding algorithm, based only on linear processing of the received signals.

The Fig. 2 shows an encoder structure for STBC. In general, a space time block code is defined by a $n_{T X P}$ transmission matrix $X$. Here $\mathrm{n}_{\mathrm{T}}$ represents the number of transmit antennas and $\mathrm{p}$ represents the number of time periods for transmission of one block of coded symbols.

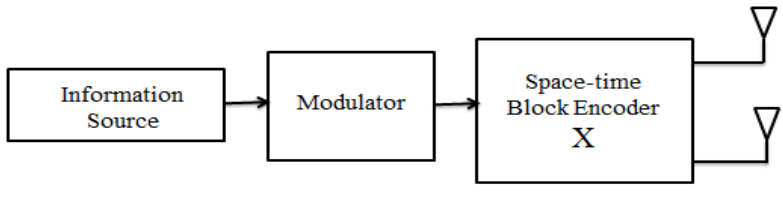

Fig.2 Encoder for STBC

\section{B. $\quad$ Space Time Block Code used in OFDM System}

The space time block codes are used in MIMO-OFDM because of its decoding simplicity. In this paper, two transmit antennas and one receive antenna is considered. Fig.3 (a) and (b) shows the transmitter \& receiver of STBC-OFDM System including space time block encoder and decoder.

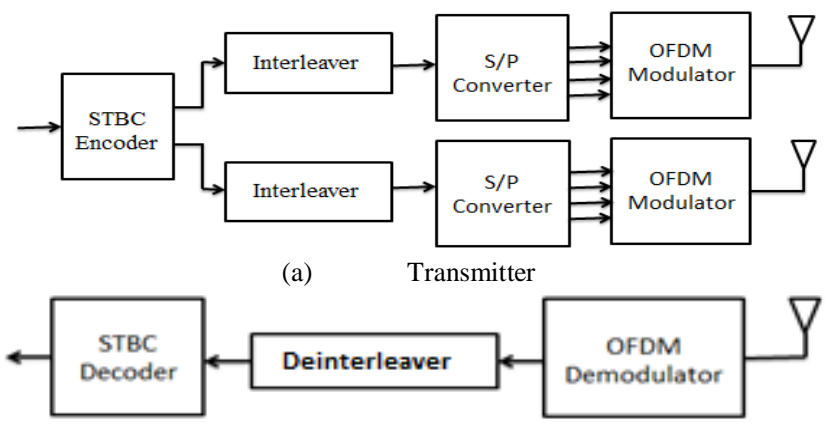

(b) Receiver

Fig.3 An STBC-OFDM system block diagram

\section{MIMO Channel Model}

In wireless communications, multipath propagation occurs because of reflection, diffraction, scattering and shadowing of the transmitted signals due to surrounding objects. As a result of this, the transmitted signals arrive at the receiver with different amplitude, different phase angles and at different time intervals. The amplitude fluctuation of the received signal is called signal fading and we assume the fading process follows a Rayleigh probability distribution function. In time domain, the channel response from the $i^{\text {th }}$ transmit antenna to the $j^{\text {th }}$ receive antenna can be given as

$$
h_{i, j}(t)=\sum_{l=0}^{L-1} \alpha_{i, j}(l) \delta\left(t-\tau_{l}\right)
$$


Where $\alpha_{i, j}(l)$ is the multi-path gain coefficient, $\mathrm{L}$ denotes the number of resolvable paths, and $\tau_{l}$ represents the path delay time of $l^{\text {th }}$ multi-path component. The frequency response of the channel is given by

$$
\begin{aligned}
& h_{i, j}^{k}(f) \\
& =\sum_{l=0}^{L-1} \alpha_{i, j}^{k}(l) e^{-j 2 \pi f \tau_{l}}
\end{aligned}
$$

Since, two transmit antennas and one receive antenna are considered, the values of $i$ are 1,2 and $\mathrm{j}$ is 1 .

\section{D. $\quad$ Statistical Models for Fading Channels}

Because of the multiplicity of factors involved in propagation in a cellular mobile environment, it is convenient to apply statistical techniques to describe signal variations. In a narrowband system, the transmitted signals usually occupy a bandwidth smaller than the channel's coherence bandwidth, which is defined as the frequency range over which the channel fading process is correlated. That is, all spectral components of the transmitted signal are subject to the same fading attenuation. This type of fading is referred to as frequency nonselective or frequency flat. In this section, Rayleigh and Rician fading models are introduced to describe signal variations in a narrowband multipath environment.

1) Rayleigh Fading: The transmission of a single tone with constant amplitude is considered. In a typical land mobile radio channel, it is assumed that the direct wave is obstructed and the mobile unit receives only reflected waves. When the number of reflected waves is large, according to the central limit theorem, two quadrature components of the received signal are uncorrelated Gaussian random processes with a zero mean and variance $\sigma_{s}^{2}$. As a result, the envelope of the received signal at any time instant undergoes a Rayleigh probability distribution and its phase obeys a uniform distribution between $-\pi$ and $\pi$. The Probability Density Function (PDF) of the Rayleigh distribution is given by

$$
p(a)=\left\{\begin{array}{cc}
\frac{a}{\sigma_{s}^{2}} \cdot e^{-a^{2} / 2 \sigma_{s}^{2}} & a \geq 0 \\
0 & a<0
\end{array}\right.
$$

2) Rician Fading: In some propagation scenarios, such as satellite or microcellular mobile radio channels, there are essentially no obstacles on the line of sight path. The received signal consists of a direct wave and a number of reflected waves. The direct wave is a stationary non-fading signal with constant amplitude. The reflected waves are independent random signals. Their sum is called the scattered component of the received signal. The sum of a constant amplitude direct signal and a Rayleigh distributed scattered signal results in a signal with a Rician envelope distribution. The PDF of the Rician distribution is given by

$$
p(a)= \begin{cases}\frac{a}{\sigma_{s}^{2}} \cdot e^{\frac{-\left(a^{2}+b^{2}\right)}{2 \sigma_{s}^{2}}} I_{0}\left(\frac{a D}{\sigma_{s}^{2}}\right) & a \geq 0 \\ 0 & a<0\end{cases}
$$

\section{Simulation Results}

Simulation results are presented to evaluate the performance of OFDM system with two transmit antennas and one receive antenna. The performance of the system is analyzed with various low SNR values ranging from $10 \mathrm{~dB}$ to $20 \mathrm{~dB}$. These Simulations are carried out for a channel Bandwidth (B.W) of $3.5 \mathrm{MHz}$ and $20 \mathrm{MHz}$ which are the lowest and highest channel B.W according to standards of 4G LTE developed by $3^{\text {rd }}$ Generation Partnership Project (3GPP).

\section{A. Performance Analysis of OFDM system without STBC} over both Rician and Rayleigh Fading channels

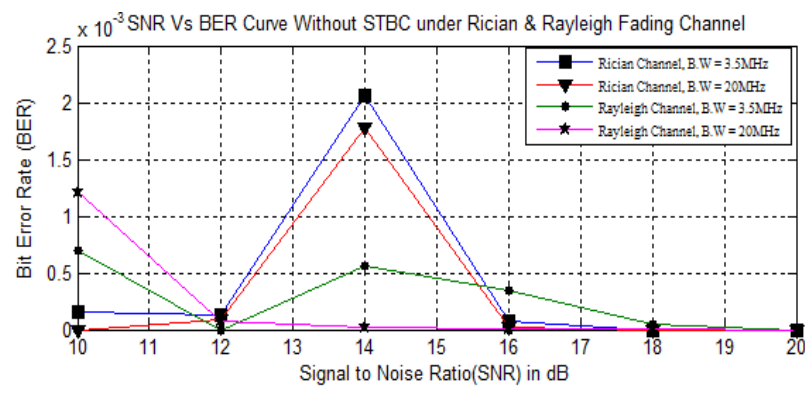

Fig.4 BER performance of OFDM System without STBC over both Rician and Rayleigh fading channels

In Fig.4, the performance analysis of OFDM system without STBC over both Rician and Rayleigh fading channels for low SNR values ranging from $10 \mathrm{~dB}$ to $20 \mathrm{~dB}$ is shown. It is observed that, for a B.W of $3.5 \mathrm{MHz}$, without Space-Time Block Codes (STBC) the Bit Error Rate (BER) is $2.058 \times 10^{-3}$ over Rician Channel, whereas over Rayleigh fading channel the BER is reduced to $5.616 \times 10^{-4}$. Even at B.W of $20 \mathrm{MHz}$, the same is be observed at a same Signal to Noise Ratio (SNR) of 14dB.

\section{B. Performance Analysis of OFDM system with STBC over both Rician and Rayleigh fading channels}

The performance of OFDM system including STBC over both Rician and Rayleigh fading Channels for low SNR values ranging from $10 \mathrm{~dB}$ to $20 \mathrm{~dB}$ is shown in the Fig.5. The BER over Rician channel for a B.W of $20 \mathrm{MHZ}$ is $1.621 \times 10^{-3}$ whereas over Rayleigh channel it has been reduced to $6.345 \times 10^{-6}$. At a same SNR of $14 \mathrm{~dB}$, the decrease in BER is observed over Rayleigh channel even for a B.W of 3.5MHZ.

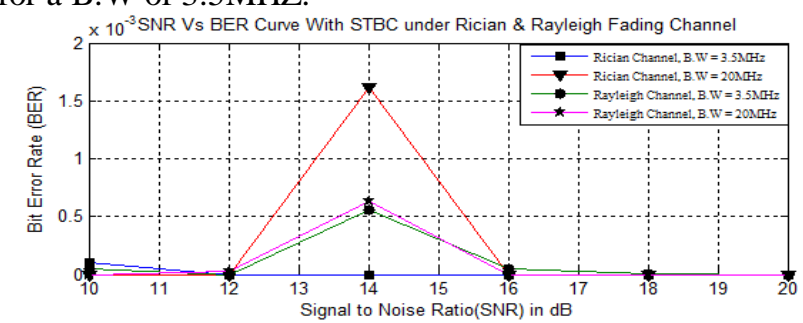

Fig.5 BER performance of OFDM System with STBC over both Rician and Rayleigh fading channels

From Fig.4 and Fig.5, it is observed that the low BER is achieved for a OFDM system under Rayleigh fading channel compared to Rician fading channel including transmit diversity techniques \& coding schemes.

\section{Performance Analysis of OFDM system both without} and with STBC over Rician fading channel

Fig. 6 presents the performance analysis of OFDM system without coding schemes \& including them over Rician 
fading channel. The system is analyzed for low SNR values ranging from $10 \mathrm{~dB}$ to $20 \mathrm{~dB}$. At channel B.W of 20MHZ, it is observed that BER without using space time block codes is $1.776 \times 10^{-3}$. Further reduction in BER of $1.621 \times 10^{-3}$ is achieved for system including Space Time Block Codes, both being simulated over Rician fading channel.

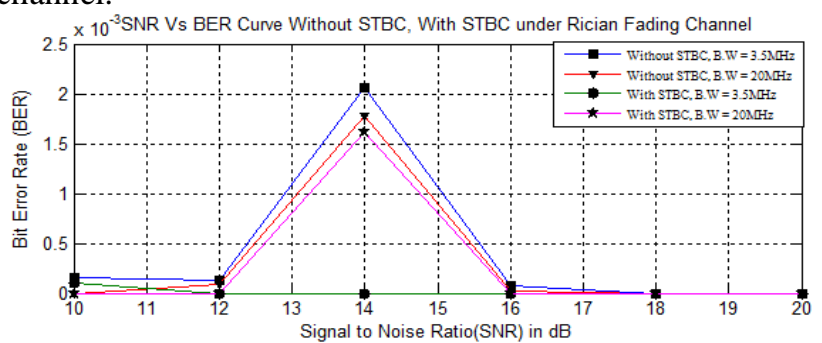

Fig.6 BER performance of OFDM System both without and with STBC over Rician fading channel

D. Performance Analysis of OFDM system both without and with STBC over Rayleigh fading channel

For low SNR values ranging from $10 \mathrm{~dB}$ to $20 \mathrm{~dB}$, the performance analysis of OFDM system without STBC and including STBC is shown in Fig.7. It is observed that, at a channel B.W of $3.5 \mathrm{MHZ}$, BER for the OFDM system without STBC is $1.834 \times 10^{-5}$ and it is reduced to $6.345 \times 10^{-}$ ${ }^{6}$ for the system including coding scheme, both being simulated over Rayleigh fading channel.

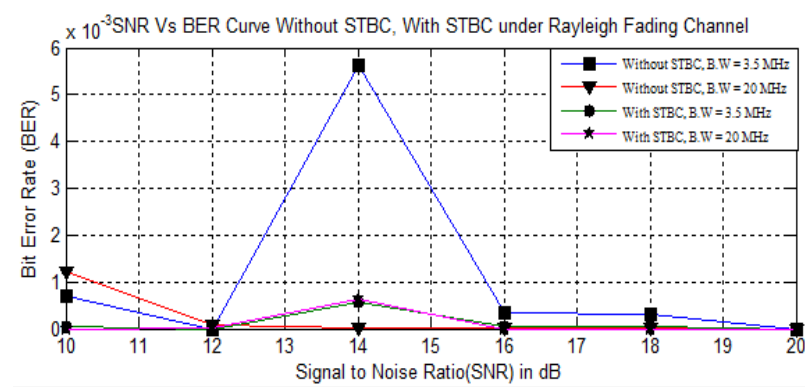

Fig.7 BER performance of OFDM System both without and with STBC over Rayleigh fading channel

From Fig.6 and Fig.7, it is observed that space time transmit diversity techniques and coding schemes effectively reduce BER. Thus system performance can be improved with high data rate and good reliability.

\section{Conclusions}

In this paper, the performance of the MIMO-OFDM system including Space Time Block Codes (STBC) over various fading channels is evaluated.

It is observed that for low SNR values ranging from $10 \mathrm{~dB}$ to $20 \mathrm{~dB}$, the Bit Error Rate (BER) for system without including STBC is $1.776 \times 10^{-3}$ and including STBC is $1.621 \times 10^{-3}$ over Rician Channel. Whereas, over Rayleigh channel with Line of Sight (LoS) the BER for system without including STBC is $1.834 \times 10^{-5}$ and including STBC it is reduced to $6.345 \times 10^{-6}$ for the same signal to Noise Ratio (SNR) of $14 \mathrm{~dB}$. As BER is reduced, the number of bits received without errors are increased.

The requirements such as high performance and high speed can thus be achieved with Space Time Block Codes (STBC). The data rate can further be improved by Differential Space Time Block Codes (DSTBC).

\section{ACKNOWLEDGEMENT}

We take immense pleasure to express our profound gratitude and heartfelt thanks to Dr.Ch.Santhi Rani, Professor, ECE for her exemplary guidance, monitoring and constant encouragement throughout the completion of our final year B.Tech project. The blessing, help and guidance given by her from time to time shall carry us a long way in the journey of life on which we are about to embark.

We also express a deep sense of gratitude to thank Dr.K.V.S.V.R.Prasad, Head of the Department, ECE and Dr.K.Surya Prakasa Rao, Principal, Daita Madhusudhana Sastry Sri Venkateswara Hindu college of Engineering, Machilipatnam for providing well organized infrastructure being helpful in completion of our project.

\section{REFERENCES}

[1] Hussain A. Alhassan, Dr. Eman Abdel Fattah, A Study on the Performance of IEEE 802.16-2004 Includes STBC, ASEE 2014 Zone I Conference, April 3-5, 2014.

[2] G. J. Foschini and M. Gans, on the limits of wireless communication in a fading environment when using multiple antennas, Wireless Personal Communication, vol. 6, pp. 311-335, March 1998.

[3] Md.Mejbaul Haque1 Mohammad Shaifur Rahman1 and Ki-Doo Kim2, Performance Analysis of MIMO-OFDM for 4G Wireless Systems under Rayleigh Fading Channel, International Journal of Multimedia and Ubiquitous Engineering, Vol. 8, No. 1, January, 2013.

[4] V. Tarokh, N. Seshadri, A. R. Calderbank, Space-Time Codes for High Data Rate Wireless Communication: Performance Criterion and Code Construction, IEEE Trans. Inform. Theory, vol. 44, no. 2, pp. 744-765, March 1998

[5] Z. Liu, G. B. Giannakis, S. Zhou, B. Muquet, Space-time coding for broadband wireless communications, Wireless Communications and Moblie Computing, vol. 1, no. 1. pp. 35-53, January 2001.

[6] J. Yuan, Z. Chen, B. Vucetic and W. Firmanto, Performance analysis of space-time coding on fading channels, in Proc. IEEE ISIT'01, Washington D.C., pp. 153, June. 2001.

[7] S. M. Alamouti, A simple transmit diversity technique for wireless communications, IEEE Journal Select. Areas Commun., vol. 16, no. 8, pp. 1451-1458, October. 1998.

[8] J. Yuan, Z. Chen, B. Vucetic and W. Firmanto, Performance analysis and design of space-time coding on fading channels, submitted to IEEE Trans. Commun.

[9] Lu, B. and Wang, X, Space-time code design in OFDM systems, IEEE Globecom, 2: Nov. 2000, 1000-4.

[10] Molisch, A. F., Win, M. Z. and winters, J. H, Space-time-frequency (STF) coding for MIMO-OFDM systems, IEEE Communications Letters, September. 2002.

[11] Lu, H.-F., Kumar, P. V. and Chung, H, On orthogonal designs and space-time codes.IEEE, International Symposium on Information Theory (ISIT), June 2002, 418.

[12] Tarokh, V., Jafarkhani, H. and Calderbank, A. R. Space-time block codes from orthogonal designs, IEEE Trans. on Information Theory, : July 1999, 1456-67.

[13] Tarokh, V., Naguib, A., Seshadri, N. and Calderbank, A. R,. Spacetime codes forhigh data rates wireless communications: performance criteria in the presence of channel estimation errors, mobility and multiple paths, IEEE Trans on Communications, February. 1999.

[14] P.Sunil Kumar, Dr.M.G.Sumithraand Ms.M.Sarumathi, Performance Comparison of Rayleigh and Rician Fading Channels in QAM modulation Scheme Using Simulink Environment, International Journal of Computational Engineering Research, Vol, 03Issue, 5.

[15] T. S. Rappaport, Wireless Communications: Principles and Practice, Prentice Hall, 1996.

[16] Ritu Gupta, Megha Kataria, Spatial Modulation Based MIMOOSDM for 4G Wireless Systems under Rayleigh Fading Channel, International Journal of Emerging Technology and Advanced Engineering, Volume 4, Issue 7, July 2014.

[17] GJ Foschini, MJ Gans, on limits of wireless communications in a fading environment when using multiple antennas, Wireless Personal Communication, pp. 311-335 (1998). 
[18] 3rd Generation Partnership Project, TS36.211 Physical Channels and Modulation. (2009).

[19] SM Alamouti, A simple transmit diversity technique for wireless communications, IEEE J Sel Areas Communication, 1451-1458 (1998).

[20] V Tarokh, H Jafarkhani, AR Calderbank, Space-time block codes from orthogonal designs.

[21] S. M. Alamouti,„A simple transmit diversity technique for wireless communications, IEEE Journal on Select Areas in Communications, vol. 16, no. 8, pp. 1451-1458, Oct. 1998.

[22] A. Vielmon, Y. Li, and J. R. Barry, Performance of Alamouti transmit diversity over time-varying Rayleigh-fading channels, IEEE Trans. On Wireless Communication. vol. 3, no. 5, pp. 13691373, September 2004.

[23] D. B. Lin, P. H. Chiang, and H. J. Li, Performance analysis of twobranch transmit diversity block-coded systems in time-varying multipath Rayleigh-fading channels, IEEE Trans. on Veh. Technol., vol. 54, no. 1, pp. 136-148, January 2005.

[24] E. Lindskog and A. Paulraj, A transmit diversity scheme for channels with intersymbol interference, in Proc. Int. Conf. Commun., New Orleans, LA, June 2002.

[25] E. Lindskog and A. Paulraj, A transmit diversity scheme for channels with intersymbol interference, in Proc. Int. Conf. Commun., New Orleans, LA, June. 2002.

[26] Hema Thota, Ch.Santhi Rani, Kiranmayee Kagitha, Anusha Balagam, Anusha Goriparthi, Naga Lakshmi Devarapalli, Performance Analysis of OFDM for 4G Wireless Systems under Various Fading Channels, International Journal Of Innovative Research In Electrical, Electronics, Instrumentation And Control Engineering, Volume 3, Issue 1, PP..51-54, January 2015

\section{BIOGRAPHIES}

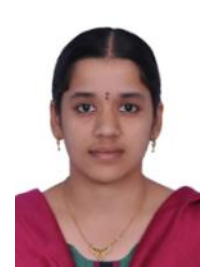

Hema Thota is pursuing B.Tech in the faculty of Electronics and Communication Engineering from D.M.S S.V.H College of Engineering, Machilipatnam, Andhra Pradesh, India. She had done a mini project titled "Digital Basic IC Tester, using only Digital ICs". She is the active member of IETE Student Forum. She secured Avvari lakshmi Narasimham Memorial Prize, Kalipatnapu Sita Mahalakshmi Memorial Prize, R.S Sastry's Prize in three consecutive years for being the topper in her B.Tech course. She had published her project work in an International Journal of Innovative Research in Electrical, Electronics, Instrumentation and Control Engineering on Wireless Communications. Her areas of research include Digital Electronics and Advanced Wireless Communications.

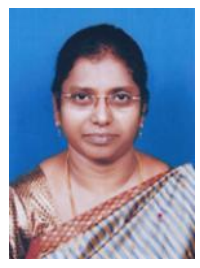

Professor Santhi Rani Chava received her B.Tech degree from Velagapudi Ramakrishna Siddhartha Engineering College, Nagarjuna University, Vijayawada, Andhra Pradesh, India. Her M.Tech degree was received from Jawaharlal Nehru Technological University College of Engineering, Ananthapur. She received Ph.D degree from Jawaharlal Nehru Technological University, Hyderabad. She had published around 24 papers in various national, international conferences and journals. She is currently working as a professor in Electronics and Communications Engineering Department, at D.M.S S.V.H College of Engineering, Machilipatnam, Andhra Pradesh, India. Her research areas include Smart Antennas, Low Power VSLI \& Advanced Wireless Communications.

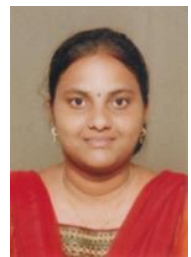

Kiranmayee Kagitha is pursuing B.Tech in the faculty of Electronics and Communication Engineering from D.M.S S.V.H College of Engineering, Machilipatnam, Andhra Pradesh, India. She secured S.N Ramachandran's prize for scoring highest marks in her first year of B.Tech from D.M.S S.V.H College of Engineering. She is the active member of IETE Student Forum. She had published her project work on Wireless Communications in an International Journal of Innovative Research in Electrical, Electronics, Instrumentation and Control Engineering. Her research area is Advanced Wireless Communications.

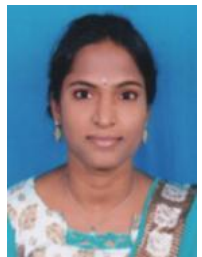

Anusha Balagam is pursuing B.Tech in Electronics and Communication Engineering from D.M.S S.V.H College of Engineering, Machilipatnam, AndhraPradesh, India. She had done a mini project titled "IR Controlled Switch". She is the member of IETE Student Forum. She had published a paper on Wireless Communications in an International Journal of Innovative Research in Electrical, Electronics, Instrumentation and Control Engineering. Her research area is Advanced Wireless Communications.

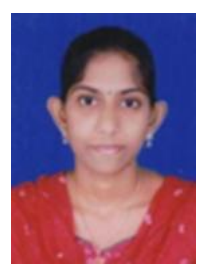

Anu sha Goriparthi is a final year B.Tech student in Electronics and Communication Engineering branch from D.M.S S.V.H College of Engineering, Machilipatnam, Andhra Pradesh, India. She had published a paper titled Performance Analysis of OFDM for 4G Wireless Systems under Various Fading Channels in an International Journal of Innovative Research in Electrical, Electronics, Instrumentation and Control Engineering in January 2015. Her research area is Advanced Wireless Communications.

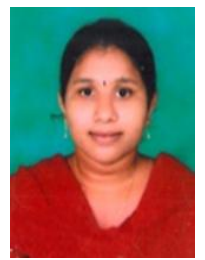

Naga Lakshmi Devarapalli is pursuing B.Tech in the faculty of Electronics and Communication Engineering from D.M.S S.V.H College of Engineering, Machilipatnam, Andhra Pradesh, India. She had published a paper titled Performance Analysis of OFDM for 4G Wireless Systems under Vari ous Fading Channels in an International Journal of Innovative Research in Electrical, Electronics, Instrumentation and Control Engineering. Her area of research is Advanced Wireless Communications. 